\title{
Speak to your Parser: \\ Interactive Text-to-SQL with Natural Language Feedback
}

\author{
Ahmed Elgohary* \\ Saghar Hosseini, Ahmed Hassan Awadallah \\ University of Maryland, College Park Microsoft Research, Redmond, WA \\ elgohary@cs.umd.edu \{sahoss, hassanam\}@microsoft.com
}

\begin{abstract}
We study the task of semantic parse correction with natural language feedback. Given a natural language utterance, most semantic parsing systems pose the problem as one-shot translation where the utterance is mapped to a corresponding logical form. In this paper, we investigate a more interactive scenario where humans can further interact with the system by providing free-form natural language feedback to correct the system when it generates an inaccurate interpretation of an initial utterance. We focus on natural language to SQL systems and construct, SPLASH, a dataset of utterances, incorrect SQL interpretations and the corresponding natural language feedback. We compare various reference models for the correction task and show that incorporating such a rich form of feedback can significantly improve the overall semantic parsing accuracy while retaining the flexibility of natural language interaction. While we estimated human correction accuracy is $81.5 \%$, our best model achieves only $25.1 \%$, which leaves a large gap for improvement in future research. SPLASH is publicly available at https:// aka.ms/Splash_dataset.
\end{abstract}

\section{Introduction}

Natural language interfaces (NLIs) have been the "holy grail" of natural language understating and human-computer interaction for decades (Woods et al., 1972; Codd, 1974; Hendrix et al., 1978; Zettlemoyer and Collins, 2005). However, early attempts in building NLIs to databases did not achieve the expected success due to limitations in language understanding capability, among other reasons (Androutsopoulos et al., 1995; Jones and Galliers, 1995). NLIs have been receiving increasing attention recently motivated by interest in developing virtual assistants, dialogue systems, and

\footnotetext{
${ }^{*}$ Most work was done while the first author was an intern at Microsoft Research.
}

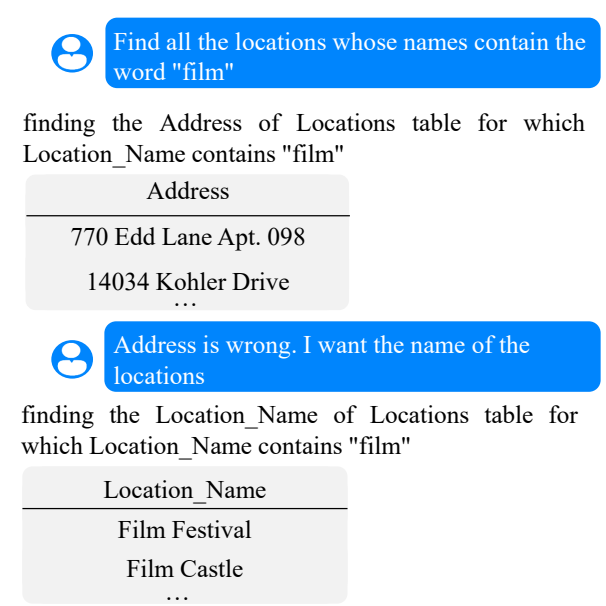

Figure 1: An example of human interaction with a Textto-SQL system to correct the interpretation of an input utterance. The system generates an initial SQL parse, explains it in natural language, and displays the execution result. Then, the system uses the human-provided natural language feedback to correct the initial parse.

semantic parsing systems. NLIs to databases were at the forefront of this wave with several studies focusing on parsing natural language utterances into an executable SQL queries (Text-to-SQL parsing).

Most of the work addressing the Text-to-SQL problem (and semantic parsing in general) frames it as a one-shot mapping problem. We establish (Section 4.1) that the majority of parsing mistakes that recent neural text-to-SQL parsers make are minor. Hence, it is often feasible for humans to detect and suggest fixes for such mistakes. Su et al. (2018) make a similar observation about parsing text to API calls (Su et al., 2017) and show that parsing mistakes could be easily corrected if humans are afforded a means of providing precise feedback. Likewise, an input utterance might be under- or mis-specified, thus extra interactions may be required to generate the desired output similarly to query refinements in information retrieval systems (Dang and Croft, 2010). 
Humans have the ability to learn new concepts or correct others based on natural language description or feedback. Similarly, previous work has explored how machines can learn from language in tasks such as playing games (Branavan et al., 2012), robot navigation (Karamcheti et al., 2017), concept learning (e.g., shape, size, etc.) classifiers (Srivastava et al., 2018), etc. Figure 1 shows an example of a text-to-SQL system that offers humans the affordance to provide feedback in natural language when the system misinterprets an input utterance. To enable this type of interactions, the system needs to: (1) provide an explanation of the underlying generated SQL, (2) provide a means for humans to provide feedback and (3) use the feedback, along with the original question, to come up with a more accurate interpretation.

In this work, we study the task of SQL parse correction with natural language feedback to enable text-to-SQL systems to seek and leverage human feedback to further improve the overall performance and user experience. Towards that goal, we make the following contributions: (1) we define the task of SQL parse correction with natural language feedback; (2) We create a framework for explaining SQL parse in natural language to allow text-to-SQL users (who may have a good idea of what kind of information resides on their databases but are not proficient in SQL Hendrix et al. (1978)) to provide feedback to correct inaccurate SQL parses; (3) we construct SPLASHSemantic Parsing with Language Assistance from Humans - a new dataset of natural language questions that a recent neural text-to-SQL parser failed to generate correct interpretation for together with corresponding human-provided natural language feedback describing how the interpretation should be corrected; and (4) we establish several baseline models for the correction task and show that the task is challenging for state-of-the-art semantic parsing models.

\section{Task}

We formally define the task of SQL parse correction with natural language feedback. Given a question $q$, a database schema $s$, a mispredicted parse $p^{\prime}$, a natural language feedback $f$ on $p^{\prime}$, the task is to generate a corrected parse $p$ (Figure 2). Following Yu et al. (2018), $s$ is defined as the set of tables, columns in each table and the primary and foreign keys of each table.

\author{
Question: \\ Find all the locations whose names contain the \\ word "film" \\ Predicted Parse: \\ SELECT AddresS FROM LOCATIONS WHERE \\ Location_Name LIKE 'ofilmo' \\ Feedback: \\ Address is wrong. I want the name of the locations \\ Gold Parse: \\ SELECT LOcation_Name FROMLOCATIONS \\ WHERE Location Name LIKE 'ofilmo' \\ Schema: \\ \begin{tabular}{|l|l|l|l|}
\hline Location_ID & Location_Name & Address & Other_Details \\
\hline
\end{tabular}
}

Figure 2: An example from our SQL parse correction task (DB Name: cre_Theme_park and Table Name: Locations). Given a question, initial predicted parse and natural language feedback on the predicted parse, the task is to predict a corrected parse that matches the gold parse.

Models are trained with tuples $q, s, p^{\prime}, f$ and gold parse $p$. At evaluation time, a model takes as input tuples in the form $q, s, p^{\prime}, f$ and hypothesizes a corrected parse $\hat{p}$. We compare $\hat{p}$ and the gold parse $p$ in terms of their exact set match (Yu et al., 2018) and report the average matching accuracy across the testing examples as the model's correction accuracy.

\section{Dataset Construction}

In this section, we describe our approach for collecting training data for the SQL parse correction task. We first generate pairs of natural language utterances and the corresponding erroneous SQL parses (Section 3.1). We then employ crowd workers (with no SQL knowledge) to provide feedback, in natural language, to correct the erroneous SQL (Section 3.3). To enable such workers to provide feedback, we show them an explanation of the generated SQL in natural language (Section 3.2). Finally, to improve the diversity of the natural language feedback, we ask a different set of annotators to paraphrase each feedback. We describe the process in detail in the remainder of this section.

\subsection{Generating Questions and Incorrect SQL Pairs}

We use the Spider dataset (Yu et al., 2018) as our source of questions. Spider has several advantages over other datasets. Compared to ATIS (Price, 


SQL:
SELECT id, name from browser GROUP
BY id ORDER BY COUNT (*) DESC
Template:
SELECT_COls_ from table_ Group
BY_COI_ORDER BY aggr__COI_
Explanation:

Step 1: Find the number of rows of each value of id in browser table.

Step 2: Find id, name of browser table with largest value in the results of step 1.

Figure 3: An example of a SQL query, the corresponding template and the generated explanation.

1990) and GeoQuery (Zelle and Mooney, 1996), Spider is much larger in scale (200 databases vs. one database, and thousands vs. hundreds of SQL parses). Compared to WikiSQL (Zhong et al., 2017), Spider questions require inducing parses of complex structures (requiring multiple tables, joining, nesting, etc.). Spider also adopts a crossdomain evaluation setup in which databases used at testing time are never seen at training time.

To generate erroneous SQL interpretations of questions in Spider, we opted for using the output of a text-to-SQL parser to ensure that our dataset reflect the actual distribution of errors that contemporary parsers make. This is a more realistic setup than artificially infusing errors in the gold SQL. We use the Seq2Struct parser (Shin, 2019) ${ }^{1}$ to generate erroneous SQL interpretations. Seq2Struct combines grammar-based decoder of Yin and Neubig (2017) with a self-attention-based schema encoding and it reaches a parsing accuracy of $42.94 \%$ on the development set of Spider. ${ }^{2}$

Note that we make no explicit dependencies on the model used for this step and hence other models could be used as well (Section 6.3).

We train Seq2Struct on $80 \%$ of Spider's training set and apply it to the remaining $20 \%$, keeping

\footnotetext{
${ }^{1}$ https://github.com/rshin/seq2struct

${ }^{2}$ When we started the dataset construction at the beginning of June 2019, we were able to achieve a parsing accuracy of $41.30 \%$ on Spider's development set which was the state-ofthe-art accuracy at the time. It is worth noting that unlike current state-of-the-art models, Seq2Struct does not use pretrained language models. It was further developed into a new model called RAT-SQL (Wang et al., 2020) which achieved a new state-of-the-art accuracy as of April 2020.
}

only cases where the generated parses do not match the gold parse (we use the exact set match of $\mathrm{Yu}$ et al. (2018)). Following the by-database splitting scheme of Spider, we repeat the 80-20 training and evaluation process for three times with different examples in the evaluation set at each run. This results in 3,183 pairs of questions and an erroneous SQL interpretation. To further increase the size of the dataset, we also ignore the top prediction in the decoder beam ${ }^{3}$ and use the following predictions. We only include cases where the difference in probability between the top and second to top SQLs is below a threshold of 0.2 . The intuition here is that those are predictions that the model was about to make and hence represent errors that the model could have made. That adds 1,192 pairs to our dataset.

\subsection{Explaining SQL}

In one of the earliest work on natural language interfaces to databases, Hendrix et al. (1978) note that many business executives, government official and other decision makers have a good idea of what kind of information residing on their databases. Yet to obtain an answer to a particular question, they cannot use the database themselves and instead need to employ the help of someone who can. As such, in order to support an interactive Text-to-SQL system, we need to be able to explain the incorrect generated SQL in a way that humans who are not proficient in SQL can understand.

We take a template-based approach to explain SQL queries in natural language. We define a template as follows: Given a SQL query, we replace literals, table and columns names and aggregation and comparison operations with generic placeholders. We also assume that all joins are inner joins (true for all Spider queries) whose join conditions are based on primary and foreign key equivalence (true for more than $96 \%$ of Spider queries). A query that consists of two subqueries combined with an intersection, union or except operations is split into two templates that are processed independently and we add an intersection/union/except part to the explanation at the end. We apply the same process to the limit operation-generate an explanation of the query without limit, then add a limit-related step at the end.

We select the most frequent 57 templates used in Spider training set which cover $85 \%$ of Spider

\footnotetext{
${ }^{3}$ We used a beam of size 20 .
} 
queries. For each SQL template, we wrote down a corresponding explanation template in the form of steps (e.g., join step, aggregation step, selection step) that we populate for each query. Figure 3 shows an example of a SQL queries, its corresponding template and generated explanations. We also implemented a set of rules for compressing the steps based on SQL semantics. For instance, an ordering step following by a "limit 1 " is replaced with "find largest/smallest" where "largest" or "smallest" is decided according to the ordering direction.

\subsection{Crowdsourcing Feedback}

We use an internal crowd-sourcing platform similar to Amazon Mechanical Turk to recruit annotators. Annotators are only selected based on their performance on other crowd-sourcing tasks and command of English. Before working on the task, annotators go through a brief set of guidelines explaining the task. ${ }^{4}$ We collect the dataset in batches of around 500 examples each. After each batch is completed, we manually review a sample of the examples submitted by each annotator and exclude those who do not provide accurate inputs from the annotators pool and redo all their annotations.

Annotators are shown the original question, the explanation of the generated SQL and asked to: (1) decide whether the generated SQL satisfies the information need in the question and (2) if not, then provide feedback in natural language. The first step is necessary since it helps identifying false negative parses (e.g., another correct parse that does not match the gold parse provided in Spider). We also use the annotations of that step to assess the extent to which our interface enables target users to interact with the underlying system. As per our assumption that target users are familiar with the kind of information that is in the database (Hendrix et al., 1978), we show the annotators an overview of the tables in the database corresponding to the question that includes the table and column names together with examples (first 2 rows) of the content. We limit the maximum feedback length to 15 tokens to encourage annotators to provide a correcting feedback based on the initial parse (that focuses on the edit to be made) rather than describing how the question should be answered.

A total of 10 annotators participated in this task. They were compensated based on an hourly rate

\footnotetext{
${ }^{4}$ We provide the data collection instructions and a screenshot of the data collection interface in the appendix.
}

\begin{tabular}{llll}
\hline Number of & Train & Dev & Test \\
\hline Examples & 7,481 & 871 & 962 \\
Databases & 111 & 9 & 20 \\
Uniq. Questions & 2,775 & 290 & 506 \\
Uniq. Wrong Parses & 2,840 & 383 & 325 \\
Uniq. Gold Parses & 1,781 & 305 & 194 \\
Uniq. Feedbacks & 7,350 & 860 & 948 \\
Feedback tokens (Avg.) & 13.9 & 13.8 & 13.1 \\
\hline
\end{tabular}

Table 1: SPLASH summary

(as opposed to per annotation) to encourage them to optimize for quality and not quantity. They took an average of 6 minutes per annotation.

To improve the diversity of the feedback we collect, we ask a separate set of annotators to generate a paraphrase of each feedback utterance. We follow the same annotators quality control measures as in the feedback collection task. An example instance from the dataset is shown in Figure 2.

\subsection{Dataset Summary}

Overall, we ask the annotators to annotate 5409 example (427 of which had the correct SQL parse and the remaining had an incorrect SQL parse). Examples with correct parse are included to test whether the annotators are able to identify correct SQL parses given their explanation and the question. Annotators are able to identify the correct parses as correct $96.4 \%$ of the time. For the examples whose predicted SQL did not match the gold SQL, annotators still marked 279 of them as correct. Upon manual examinations, we found that annotators were indeed correct in doing so $95.5 \%$ of the time. Even though the predicted and gold SQLs did not match exactly, they were equivalent (e.g., 'price between 10 and 20' vs. $'$ price $\geq 10$ and price $\leq 20^{\prime}$ ).

After paraphrasing, we ended up with 9,314 question-feedback pairs, 8352 of which correspond to questions in the Spider training split and 962 from the spider development split. We use all the data from the Spider development split as our test data. We hold out $10 \%$ of the remaining data (split by database) to use as our development set and use the rest as the training set. Table 1 provides a summary of the final dataset.

\section{Dataset Analysis}

We conduct a more thorough analysis of SPLASH in this section. We study the characteristics of the mistakes made by the parser as well as characteristics of the natural language feedback. 


\subsection{Error Characteristics}

We start by characterizing the nature of errors usually made by the models in parsing the original utterance to SQL. To understand the relation between the gold and the predicted SQL, we measure the edit distance between them for all cases for which the model made a mistake in the SQL prediction. We measure the edit distance by the number of edit segments (delete, insert, replace) between both parses. We find the minimal sequence of tokenlevel edits using the levenshtein distance algorithm. Then, we combine edits of the same type (delete, insert, replace) applied to consecutive positions in the predicted parse in one segment. Figure 4 shows a frequency histogram of different values of edit distance. We observe that most inaccurate predictions lie within a short distance from the correct SQL ( $78 \%+$ within a distance of 3 or less).

In addition to the number of edits, we also characterize the types of edits needed to convert the predicted SQL to the gold one. Our edit distance calculations support three operations replace, insert and delete. Those correspond to 58\%,,31\% and $11 \%$ of the edit operations respectively. Most of the edits are rather simple and require replacing, inserting or deleting a single token ( $68.1 \%$ of the edits). The vast majority of those correspond to editing a schema item (table or column name): $89.2 \%$, a SQL keyword (e.g., order direction, aggregation, count, distinct, etc.): $7.4 \%$, an operator (greater than, less than, etc.): $2.2 \%$ or a number (e.g. for a limit operator): $1.2 \%$.

The edits between the predicted and generated SQL spanned multiple SQL keywords. The distribution of different SQL keywords appearing in edits and their distribution across edit types (replace, insert or delete) is shown in Figure 5. Note that a single edit could involve multiple keywords (e.g., multiple joins, a join and a where clause, etc.). Interestingly, many of the edits involve a join highlighting that handling utterances that require a join is harder and more error prone. Following join, most edits involve where clauses (making the query more or less specific), aggregation operators, counting and selecting unique values.

The error analysis demonstrates that many of the errors made by the model are in fact not significant and hence it is reasonable to seek human feedback to correct them.

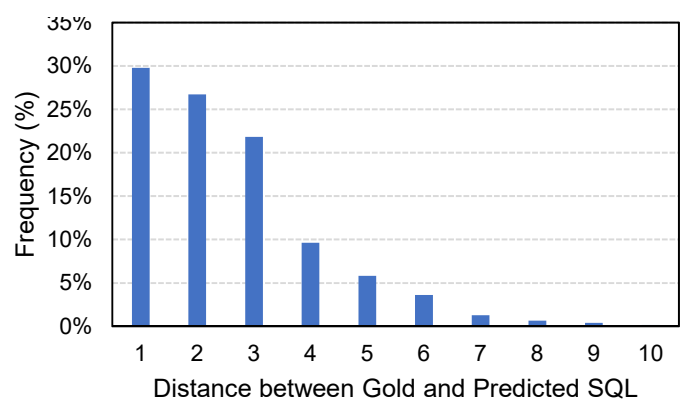

Figure 4: A histogram of the distance between the gold and the predicted SQL.

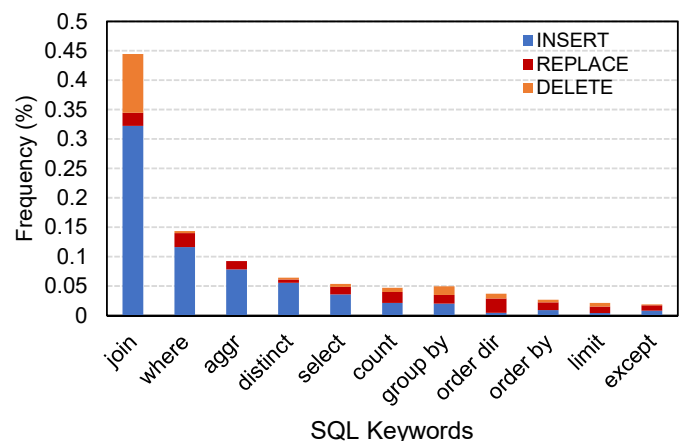

Figure 5: A histogram of different SQL keywords appearing in edits (between the gold and predicted SQL) and their distribution across edit types (replace, insert or delete).

\subsection{Feedback Characteristics}

To better understand the different types of feedback our annotators provided, we sample 200 examples from the dataset, and annotate them with the type of the feedback. We start by assigning the feedback to one of three categories: (1) Complete: the feedback fully describes how the predicted SQL can be corrected , (2) Partial: the feedback describes a way to correct the predicted SQL but only partially and (3) Paraphrase: the feedback is a paraphrase of the original question. The sample had $81.5 \%$ for Complete, $13.5 \%$ for Partial and 5.0\% for Paraphrase feedback. Examples of each type of feedback are shown in Table 2. Upon further inspection of the partial and paraphrase feedback, we observe that they mostly happen when the distance between the predicted and gold SQL is high (major parsing errors). As such, annotators opt for providing partial feedback (that would at least correct some of the mistakes) or decide to rewrite the question in a different way.

We also annotate and present the types of feedback, in terms of changes the feedback is suggesting, in Table 3. Note that the same feedback may suggest multiple changes at the same time. The 


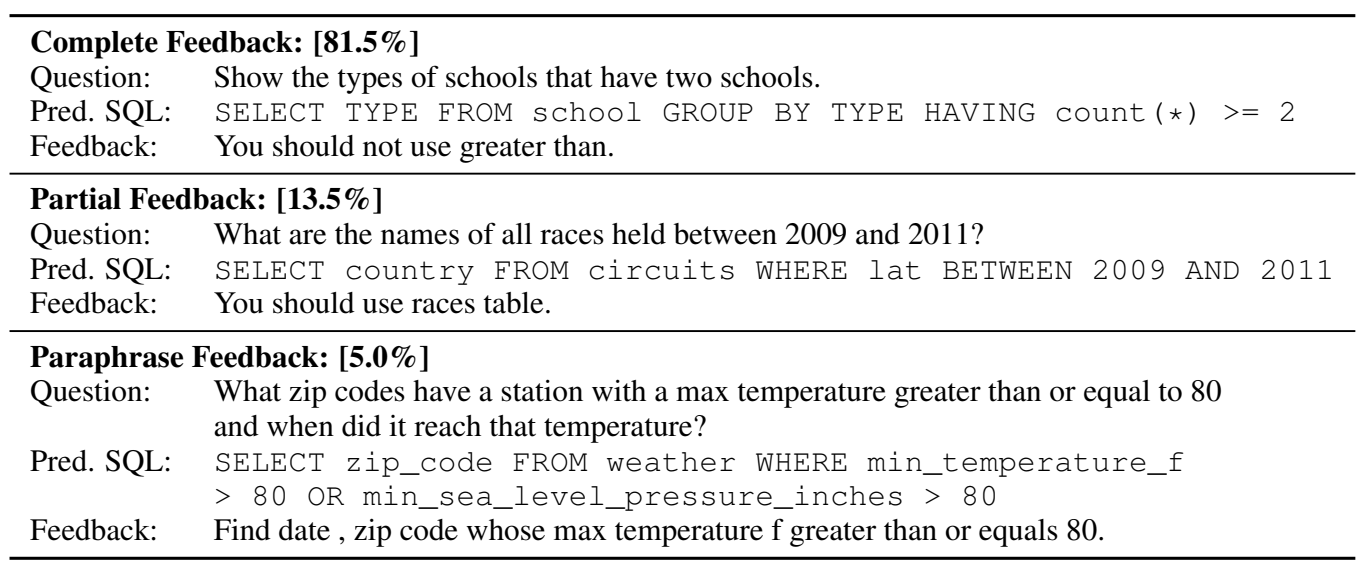

Table 2: Examples (question, predicted SQL and feedback) of complete, partial and paraphrase feedback

table shows that the feedback covers a broad range of types, which matches our initial analysis of error types. We find that a majority of feedback is referencing the retrieved information. In many such cases, the correct information has not been retrieved because the corresponding table was not used in the query. This typically corresponds to a missing inner one-to-one join operation and agrees with our earlier analysis on edit distance between the gold and predicted SQL. The second most popular category is incorrect conditions or filters followed by aggregation and ordering errors. We split the first two categories by whether the information/conditions are missing, need to be replaced or need to be removed. We observe that most of the time the information or condition needs to be replaced. This is followed by missing information that needs to be inserted and then unnecessary ones that need to be removed.

We heuristically identify feedback patterns for each collected feedback. To identify the feedback pattern, we first locate the central predicate in the feedback sentence using a semantic role labeler (He et al., 2015). Since some feedback sentences can be broken into multiple sentence fragments, a single feedback may contain more than one central predicate. For each predicate, we identify its main arguments. We represent every argument with its first non stopword token. To reduce the vocabulary, we heuristically identify column mentions and replace them with the token 'item'.

We visualize the distribution of feedback patterns for the top 60 most frequent patterns in Figure 6 , and label the ones shared among multiple patterns. As is shown, our dataset covers a diverse variety of feedback patterns centered around key operations to edit the predicted SQL that reference

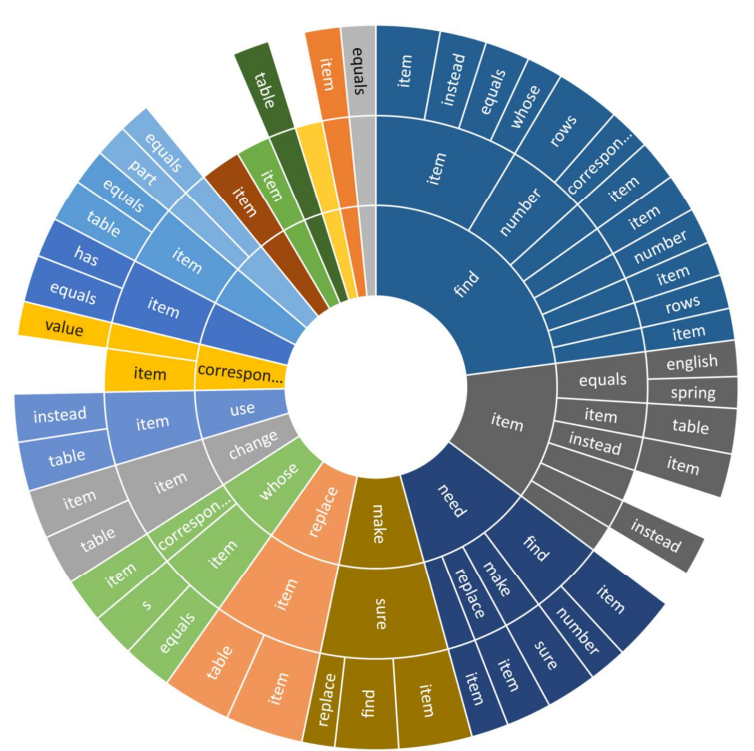

Figure 6: Patterns of feedback covered in our dataset. Patterns are extracted heuristically using predicates and arguments extracted from the feedback sentence. The figure shows the top 60 frequent patterns.

operations, column names and values.

\section{Related Work}

Our work is linked to multiple existing research lines including semantic parsing, learning through interaction (Li et al., 2017a; Hancock et al., 2019; Li et al., 2017b, inter alia) and learning from natural language supervision (Srivastava et al., 2017; CoReyes et al., 2019; Srivastava et al., 2018; Hancock et al., 2018; Ling and Fidler, 2017, inter alia). We discuss connections to the most relevant works.

Text-to-SQL Parsing: Natural language to SQL (natural language interfaces to databases) has been an active field of study for several decades (Woods et al., 1972; Hendrix et al., 1978; Warren and Pereira, 1982; Popescu et al., 2003; Li 


\begin{tabular}{lll}
\hline Feedback Type & $\%$ & Example \\
\hline $\begin{array}{l}\text { Information } \\
\text { - Missing }\end{array}$ & $13 \%$ & I also need the number of different services \\
- Wrong & $36 \%$ & Return capacity in place of height \\
- Unnecessary & $4 \%$ & No need to return email address \\
Conditions & & \\
- Missing & $10 \%$ & ensure they are FDA approved \\
- Wrong & $19 \%$ & need to filter on open year not register year \\
- Unnecessary & $7 \%$ & return results for all majors \\
Aggregation & $6 \%$ & I wanted the smallest ones not the largest \\
Order/Uniq & $5 \%$ & only return unique values \\
\hline
\end{tabular}

Table 3: Examples of feedback annotators provided for different types

and Jagadish, 2014). This line of work has been receiving increased attention recently driven, in part, by the development of new large scale datasets such as WikiSQL (Zhong et al., 2017) and Spider (Yu et al., 2018). The majority of this work has focused on mapping a single query to the corresponding SQL with the exception of a few datasets, e.g., SParC (Yu et al., 2019b) and CoSQL (Yu et al., 2019a), that target inducing SQL parses for sequentially related questions. While these datasets focus on modeling conversational dependencies between questions, SPLASH evaluates the extent to which models can interpret and apply feedback on the generated parses. We empirically confirm that distinction in Section 6.3.

Learning from Feedback: Various efforts have tried to improve semantic parsers based on feedback or execution validation signals. For example, Clarke et al. (2010) and Artzi and Zettlemoyer (2013) show that semantic parsers can be improved by learning from binary correct/incorrect feedback signals or validation functions. Iyer et al. (2017) improve text-to-SQL parsing by counting on humans to assess the correctness of the execution results generated by the inferred parses. In their system, parses with correct results are used to augment the training set together with crowdsourced gold parses of the parses that are marked as incorrect. Lawrence and Riezler (2018) show that a text-to-Overpass parser can be improved using historic logs of token-level binary feedback (collected using a graphical user interface that maps an Overpass query to predefined blocks) on generated parses. We note that our work is different from this line of work in that we do not seek to retrain and generally improve the parser, rather we focus on the task of immediately incorporating the natural language feedback to correct an initial parse.
Interactive Semantic Parsing Multiple other efforts sought to interactively involve humans in the parsing process itself. He et al. (2016) ask simplified questions about uncertain dependencies in CCG parses and use the answers as soft constraints to regenerate the parse. Both $\mathrm{Li}$ and Jagadish (2014) and Su et al. (2018) generate semantic parses and present them in a graphical user interface that humans can control to edit the initial parse. Gur et al. (2018) ask specific predefined multiple choice questions about a narrow set of predefined parsing errors. This interaction model together with the synthetically generated erroneous parses that are used for training can be appropriate for simple text-to-SQL parsing instance as in WikiSQL, which was the only dataset used for evaluation. Yao et al. (2019b) ask yes/no questions about the presence of SQL components while generating a SQL parse one component at a time. Our work falls under the general category of interactive semantic parsing. However, our interaction model is solely based on natural language feedback which can convey richer information and offering a more flexible interaction. Our work is closest to (Labutov et al., 2018), which also studies correcting semantic parses with natural language feedback, but we (1) focus on text-to-SQL parsing and build on a multi-domain dataset that requires generating complex semantic structures and generalizing to unseen domains (Labutov et al. consider only the domain of email and biographical research); (2) pair the mispredicted parses and feedback with gold parses ${ }^{5}$ in both our training and testing splits which benefits a wider class of correction models; and (3) show that incorporating the mispredicted parse significantly improves the correction accu-

\footnotetext{
${ }^{5}$ In real world scenarios, the gold parse is the final parse that the user approves after a round (or more) of corrections.
} 
racy (on the contrary to the findings of Labutov et al.).

Asking Clarifying Questions: Another relevant research direction focused on extending semantic parsers beyond one-shot interactions by creating agents that can ask clarifying questions that resolve ambiguities with the original question. For example, Yao et al. (2019a) showed that using reinforcement learning based agents that can ask clarifying questions can improve the performance of semantic parsers in the "If-Then recipes" domain. Generating clarifying questions have been studied in multiple domains to resolve ambiguity caused by speech recognition failure (Stoyanchev et al., 2014), recover missing information in question answering (Rao and Daumé III, 2018) or clarify information needs in open-domain informationseeking (Aliannejadi et al., 2019). Our work is different from this research in that we focus on enabling and leveraging human feedback that corrects an initial parse of a fully specified question rather than spotting and clarifying ambiguities.

\section{Experiments}

We present and evaluate a set of baseline models for the correction task (Section 2) in which we use SPLASH for training and testing (unless otherwise stated). Our main evaluation measure is the correction accuracy - the percentage of the testing set examples that are corrected-in which we follow Yu et al. (2018) and compare the corrected parse to the gold parse using exact set match. ${ }^{6}$ We also report the end-to-end accuracy on Spider development set (which we use to construct our testing set) of the two turn interaction scenario: first Seq2Struct attempts to parse the input question. If it produced a wrong parse the question together with that parse and the corresponding feedback are attempted using the correction model. An example is considered "correct" if any of the two attempts produces the correct parse. 7

\subsection{Baselines}

Methods that ignore the feedback: One approach for parse correction is re-ranking the decoder beam (top- $n$ predictions) (Yin and Neubig,

\footnotetext{
${ }^{6}$ Exact set match is a binary measure of exact string matching between SQL queries that handles ordering issues.

7 Seq2Struct produces correct parses for 427/1034 of Spider Dev. 511 of the remaining examples are supported by our SQL explanation patterns. We estimate the end-to-end accuracy as $(427+511 * X / 100) / 1034$, where $X$ is the correction accuracy.
}

2019). Here, we simply discard the top-1 candidate and sample uniformly and with probabilities proportional to the parser score of each candidate. We also report the accuracy of deterministically choosing the second candidate.

Handcrafted re-ranking with feedback: By definition, the feedback $f$ describes how to edit the initial parse $p^{\prime}$ to reach the correct parse. We represent the "diff" between $p^{\prime}$ and each candidate parse in the beam $p_{i}$ as set of schema items that appear only in one of them. For example, the diff between select first_name, last_name from students and select first_name from teachers is \{last_name, students, teachers $\}$. We assign a score to $p_{i}$ equals to the number of matched schema items in the diff with $f$. A schema item (e.g., "first_name") is considered to be mentioned in $f$ is all the individual tokens ("first" and "name") are tokens in $f$.

Seq2Struct+Feedback: The Seq2Struct model we use to generate erroneous parses for data collection (Section 3.1) reached an accuracy of $41.3 \%$ on Spider's development set when trained on the full Spider training set for 40,000 steps. After that initial training phase, we adapt the model to incorporating the feedback by appending the feedback to the question for each training example in SPLASH and we continue training the model to predict the gold parse for another 40,000 steps. We note that Seq2Struct+Feedback does not use the mispredicted parses.

EditSQL+Feedback: EditSQL (Zhang et al., 2019) is the current state-of-the-art model for conversational text-to-SQL. It generates a parse for an utterance at a conversation turn $i$ by editing (i.e., copying from) the parse generated at turn $i-1$ while condition on all previous utterances as well as the schema. We adapt EditSQL for the correction task by providing the question and the feedback as the utterances at turn one and two respectively, and we force it to use the mispredicted parse the the prediction of turn one (rather than predicting it). We train the model on the combination of the training sets of SPLASH and Spider (which is viewed as single turn conversations). ${ }^{8}$

To provide an estimate of human performance, we report the percentage of feedback instances la-

\footnotetext{
${ }^{8}$ We exclude turn one predictions from the training loss when processing SPLASH examples otherwise, the model would be optimized to produce the mispredicted parses. We use the default hyper-parameters provided by the authors together with the development set of SPLASH for early stopping.
} 


\begin{tabular}{lcc}
\hline & \multicolumn{2}{c}{ Exact Match Accuracy (\%) } \\
Baseline & Norrection & End-to-End \\
\hline $\begin{array}{l}\text { Without Feedback } \\
\quad \Rightarrow \text { Seq2Struct }\end{array}$ & 41.30 \\
$\quad \Rightarrow$ Re-ranking: Uniform & 2.39 & 42.48 \\
$\quad \Rightarrow$ Re-ranking: Parser score & 11.26 & 46.86 \\
$\quad \Rightarrow$ Re-ranking: Second Best & 11.85 & 47.15 \\
With Feedback & & \\
$\quad \Rightarrow$ Re-ranking: Handcrafted & 16.63 & 49.51 \\
$\quad \Rightarrow$ Seq2Struct+Feedback & 13.72 & 48.08 \\
$\quad \Rightarrow$ EditSQL+Feedback & $\mathbf{2 5 . 1 6}$ & $\mathbf{5 3 . 7 3}$ \\
\hline Re-ranking Upper Bound & 36.38 & 59.27 \\
Estimated Human Accuracy & 81.50 & 81.57 \\
\hline
\end{tabular}

Table 4: Correction and End-to-End accuracies of Baseline models.

beled as Complete as described in Section 4.2. We also report the re-ranking upper bound (the percentage of test examples whose gold parses exist in Seq2Struct beam).

\subsection{Main Results}

The results in Table 4 suggest that: (1) the feedback we collect is indeed useful for correcting erroneous parses; (2) incorporating the mispredicted parse helps the correction process (even a simple handcrafted baseline that uses the mispredicted parases outperforms a strong trained neural model); and (3) the state-of-the-art EditSQL model equipped with BERT (Devlin et al., 2019) achieves the best performance, yet it still struggles with the task we introduce, leaving a large gap for improvement.

\subsection{Analysis}

Does EditSQL+Feedback use the feedback? To confirm that EditSQL+Feedback does not learn to ignore the feedback, we create a test set of random feedback by shuffling the feedback of SPLASH test examples. The accuracy on the randomized test set drops to $5.6 \%$.

Is SPLASH just another conversational textto-SQL dataset? We evaluate the trained EditSQL models on SParC and CoSQL (state-of-the-art models trained by EditSQL authors) on SPLASH test set, and we get accuracies of $3.4 \%$ and $3.2 \%$, respectively. That confirms that SPLASH targets different modeling aspects as we discuss in Section 5.

Is SPLASH only useful for correcting Seq2Struct errors? EditSQL is also shown to achieve strong results on Spider $(57.6 \%$ on the development set) when used in a single-turn mode (state-of-the-art when we started writing this paper). We collect feedback for a sample of 179 mispredicted parses produces by EditSQL. ${ }^{9}$ Using the EditSQL+Feedback model trained on SPLASH we get a correction accuracy of $14.6 \%$ for EditSQL errors.

\section{Conclusions and Future Work}

We introduce the task of SQL parse correction using natural language feedback together with a dataset of human-authored feedback paired with mispredicted and gold parses. We compare baseline models and show that natural language feedback is effective for correcting parses, but still stateof-the-art models struggle to solve the task. Future work can explore improving the correction models, leveraging logs of natural language feedback to improve text-to-SQL parsers, and expanding the dataset to include multiple turns of correction.

\section{Acknowledgments}

We thank our ACL reviewers for their feedback and suggestions. Ahmed Elgohary completed part of this work while being supported by a grant from the Defense Advanced Research Projects Agency and Air Force Research Laboratory, and awarded to Raytheon BBN Technologies under contract number FA865018-C-7885.

\footnotetext{
${ }^{9}$ We started with 200 , but 21 of them turned out to have alternative correct parses (false negatives).
} 


\section{References}

Mohammad Aliannejadi, Hamed Zamani, Fabio Crestani, and W. Bruce Croft. 2019. Asking clarifying questions in open-domain information-seeking conversations. In Proceedings of the ACM SIGIR Conference on Research and Development in Information Retrieval.

Ion Androutsopoulos, Graeme D Ritchie, and Peter Thanisch. 1995. Natural language interfaces to databases-an introduction. Natural language engineering, 1.

Yoav Artzi and Luke Zettlemoyer. 2013. Weakly supervised learning of semantic parsers for mapping instructions to actions. Transactions of the Association for Computational Linguistics.

SRK Branavan, David Silver, and Regina Barzilay. 2012. Learning to win by reading manuals in a monte-carlo framework. Journal of Artificial Intelligence Research, 43.

James Clarke, Dan Goldwasser, Ming-Wei Chang, and Dan Roth. 2010. Driving semantic parsing from the world's response. In Conference on Computational Natural Language Learning.

John D Co-Reyes, Abhishek Gupta, Suvansh Sanjeev, Nick Altieri, John DeNero, Pieter Abbeel, and Sergey Levine. 2019. Meta-learning languageguided policy learning. In Proceedings of the International Conference on Learning Representations.

Edgar F Codd. 1974. Seven steps to rendezvous with the casual user. IBM Corporation.

Van Dang and Bruce W Croft. 2010. Query reformulation using anchor text. In Proceedings of ACM International Conference on Web Search and Data Mining.

Jacob Devlin, Ming-Wei Chang, Kenton Lee, and Kristina Toutanova. 2019. BERT: Pre-training of deep bidirectional transformers for language understanding. In Conference of the North American Chapter of the Association for Computational Linguistics.

Izzeddin Gur, Semih Yavuz, Yu Su, and Xifeng Yan. 2018. DialSQL: Dialogue based structured query generation. In Proceedings of the Association for Computational Linguistics.

Braden Hancock, Antoine Bordes, Pierre-Emmanuel Mazare, and Jason Weston. 2019. Learning from dialogue after deployment: Feed yourself, chatbot! In Proceedings of the Association for Computational Linguistics.

Braden Hancock, Paroma Varma, Stephanie Wang, Martin Bringmann, Percy Liang, and Christopher Ré. 2018. Training classifiers with natural language explanations. In Proceedings of the Association for Computational Linguistics.
Luheng He, Mike Lewis, and Luke Zettlemoyer. 2015. Question-answer driven semantic role labeling: Using natural language to annotate natural language. In Proceedings of Empirical Methods in Natural Language Processing.

Luheng He, Julian Michael, Mike Lewis, and Luke Zettlemoyer. 2016. Human-in-the-loop parsing. In Proceedings of Empirical Methods in Natural Language Processing.

Gary G Hendrix, Earl D Sacerdoti, Daniel Sagalowicz, and Jonathan Slocum. 1978. Developing a natural language interface to complex data. ACM Transactions on Database Systems, 3.

Srinivasan Iyer, Ioannis Konstas, Alvin Cheung, Jayant Krishnamurthy, and Luke Zettlemoyer. 2017. Learning a neural semantic parser from user feedback. In Proceedings of the Association for Computational Linguistics.

Karen Sparck Jones and Julia R Galliers. 1995. Evaluating natural language processing systems: An analysis and review, volume 1083.

Siddharth Karamcheti, Edward Clem Williams, Dilip Arumugam, Mina Rhee, Nakul Gopalan, Lawson L.S. Wong, and Stefanie Tellex. 2017. A tale of two DRAGGNs: A hybrid approach for interpreting action-oriented and goal-oriented instructions. In Proceedings of the First Workshop on Language Grounding for Robotics.

Igor Labutov, Bishan Yang, and Tom Mitchell. 2018. Learning to learn semantic parsers from natural language supervision. In Proceedings of Empirical Methods in Natural Language Processing.

Carolin Lawrence and Stefan Riezler. 2018. Improving a neural semantic parser by counterfactual learning from human bandit feedback. In Proceedings of the Association for Computational Linguistics.

Fei Li and HV Jagadish. 2014. Constructing an interactive natural language interface for relational databases. In Proceedings of the VLDB Endowment.

Jiwei Li, Alexander H. Miller, Sumit Chopra, Marc'Aurelio Ranzato, and Jason Weston. 2017a. Dialogue learning with human-in-the-loop. In Proceedings of the International Conference on Learning Representations.

Jiwei Li, Alexander H. Miller, Sumit Chopra, Marc'Aurelio Ranzato, and Jason Weston. 2017b. Learning through dialogue interactions by asking questions. In Proceedings of the International Conference on Learning Representations.

Huan Ling and Sanja Fidler. 2017. Teaching machines to describe images via natural language feedback. In Proceedings of Advances in Neural Information Processing Systems. 
Ana-Maria Popescu, Oren Etzioni, and Henry Kautz. 2003. Towards a theory of natural language interfaces to databases. In International Conference on Intelligent User Interfaces.

P. J. Price. 1990. Evaluation of spoken language systems: the ATIS domain. In Speech and Natural Language: Proceedings of a Workshop Held at Hidden Valley, Pennsylvania.

Sudha Rao and Hal Daumé III. 2018. Learning to ask good questions: Ranking clarification questions using neural expected value of perfect information. In Proceedings of the Association for Computational Linguistics.

Richard Shin. 2019. Encoding database schemas with relation-aware self-attention for text-to-sql parsers. arXiv preprint arXiv:1906.11790.

Shashank Srivastava, Igor Labutov, and Tom Mitchell. 2017. Joint concept learning and semantic parsing from natural language explanations. In Proceedings of Empirical Methods in Natural Language Processing.

Shashank Srivastava, Igor Labutov, and Tom Mitchell. 2018. Zero-shot learning of classifiers from natural language quantification. In Proceedings of the Association for Computational Linguistics.

Svetlana Stoyanchev, Alex Liu, and Julia Hirschberg. 2014. Towards natural clarification questions in dialogue systems.

Yu Su, Ahmed Hassan Awadallah, Madian Khabsa, Patrick Pantel, and Michael Gamon. 2017. Building natural language interfaces to web apis. In Proceedings of the ACM International Conference on Information and Knowledge Management.

Yu Su, Ahmed Hassan Awadallah, Miaosen Wang, and Ryen W. White. 2018. Natural language interfaces with fine-grained user interaction: A case study on web apis. In Proceedings of the ACM SIGIR Conference on Research and Development in Information Retrieval.

Bailin Wang, Richard Shin, Xiaodong Liu, Oleksandr Polozov, and Matthew Richardson. 2020. RATSQL: Relation-aware schema encoding and linking for text-to-sql parsers. In Proceedings of the Association for Computational Linguistics.

David H.D. Warren and Fernando C.N. Pereira. 1982. An efficient easily adaptable system for interpreting natural language queries. American Journal of Computational Linguistics, 8.

W. A. Woods, Ronald M Kaplan, and Bonnie L. Webber. 1972. The lunar sciences natural language information system: Final report. BBN Report 2378.

Ziyu Yao, Xiujun Li, Jianfeng Gao, Brian Sadler, and Huan Sun. 2019a. Interactive semantic parsing for if-then recipes via hierarchical reinforcement learning. In Association for the Advancement of Artificial Intelligence.

Ziyu Yao, Yu Su, Huan Sun, and Wen-tau Yih. 2019b. Model-based interactive semantic parsing: A unified framework and a text-to-SQL case study. In Proceedings of Empirical Methods in Natural Language Processing.

Pengcheng Yin and Graham Neubig. 2017. A syntactic neural model for general-purpose code generation. In Proceedings of the Association for Computational Linguistics.

Pengcheng Yin and Graham Neubig. 2019. Reranking for neural semantic parsing. In Proceedings of the Association for Computational Linguistics.

Tao Yu, Rui Zhang, He Yang Er, Suyi Li, Eric Xue, Bo Pang, Xi Victoria Lin, Yi Chern Tan, Tianze Shi, Zihan Li, et al. 2019a. CoSQL: A conversational text-to-sql challenge towards cross-domain natural language interfaces to databases. In Proceedings of Empirical Methods in Natural Language Processing.

Tao Yu, Rui Zhang, Kai Yang, Michihiro Yasunaga, Dongxu Wang, Zifan Li, James Ma, Irene Li, Qingning Yao, Shanelle Roman, Zilin Zhang, and Dragomir Radev. 2018. Spider: A largescale human-labeled dataset for complex and crossdomain semantic parsing and text-to-SQL task. In Proceedings of Empirical Methods in Natural Language Processing.

Tao Yu, Rui Zhang, Michihiro Yasunaga, Yi Chern Tan, Xi Victoria Lin, Suyi Li, Heyang Er, Irene Li, Bo Pang, Tao Chen, et al. 2019b. SParC: Crossdomain semantic parsing in context. In Proceedings of the Association for Computational Linguistics.

John M. Zelle and Raymond J. Mooney. 1996. Learning to parse database queries using inductive logic programming. In Association for the Advancement of Artificial Intelligence.

Luke Zettlemoyer and Michael Collins. 2005. Learning to map sentences to logical form: Structured classiication with probabilistic categorial grammars. In Proceedings of Uncertainty in Artificial Intelligence.

Rui Zhang, Tao Yu, He Yang Er, Sungrok Shim, Eric Xue, Xi Victoria Lin, Tianze Shi, Caiming Xiong, Richard Socher, and Dragomir Radev. 2019. Editing-based sql query generation for cross-domain context-dependent questions. In Proceedings of Empirical Methods in Natural Language Processing.

Victor Zhong, Caiming Xiong, and Richard Socher. 2017. Seq2SQL: Generating structured queries from natural language using reinforcement learning. arXiv preprint arXiv:1709.00103. 


\section{A Appendix}

\section{A.1 Feedback Collection instructions}

Figure 7 shows the instructions shown to the annotators.

\section{A.2 Feedback Collection Interface Screenshot}

Figure 8 shows an example of the data collection interface. The Predicted SQL is: 'SELECT

name, salary FROM instructor

WHERE dept_name LIKE "\%math\%"'.

Note that neither the gold nor the predicted SQL are shown to the annotator.

\section{A.3 Example of Explanations}

Figure 9 shows several examples of how different SQL components can be explained in natural language.

\section{Correcting Steps for Answering Questions.}

1. We have some information stored in tables; each row is a record that consists of one or more columns. Using the given tables, we can answer questions by doing simple systematic processing steps over the tables. Notice that the answer to the question is always the result of the last step. Also, notice that the steps might not be in perfect English as they were generated automatically. Each step, generates a table of some form.

2. For each question, we have generated steps to answer it, but it turned out that something is wrong with the steps. Your task is write down in English a short (one sentence most of the time) description of the mistakes and how it can be correct. It is important to note that we are not looking for rewritings of steps, but rather we want to get short natural English commands (15 words at most) that describes the correction to be made to get the correct answer.

3. Use proper and fluent English. Don't use math symbols.

4. Don't rewrite the steps after correcting them. But rather, just describe briefly the change that needs to be made.

5. We show only two example values from each table to help you understand the contents of each table. Tables typically contain several rows. Never use the shown values to write your input.

6. There could be more than one wrong piece in the steps. Please, make sure to mention all of them not just one.

7. If the steps are correct, just check the "All steps are correct" box

8. Some of the mistakes are due to additional steps or parts of steps. Your feedback can suggest removing those parts.

9. Do not just copy parts of the questions. Be precise in your input.

10. If in doubt about how to correct a mistake, just mention what is wrong.

11. You do not have to mention which steps contain mistakes. If in doubt, do not refer to a particular step.

12. The generated steps might not sound like the smartest way for answering the question. But it is the most systematic way that works for all kinds of questions and all kinds of tables. Please, do not try to propose smarter steps.

Figure 7: Crowd-sourcing instructions 


\section{Question:}

Find the name and salary of instructors who are advisors of the students from the Math department.

\section{Steps:}

find the name, salary of instructor table for which dept_name equals Math

\section{Tables with example values:}

instructor

\begin{tabular}{|c|c||c|c|}
\hline ID & name & dept_name & salary \\
\hline 65931 & Pimenta & Cybernetics & 79866.95 \\
\hline 28400 & Atanassov & Statistics & 84982.92 \\
\hline
\end{tabular}

\section{student}

\begin{tabular}{|c|c|c|c|}
\hline ID & name & dept_name & tot_cred \\
\hline 32245 & Saariluoma & Statistics & 12 \\
\hline 79589 & Schopp & Elec. Eng. & 104 \\
\hline
\end{tabular}

\section{Feedback:}

\section{All steps are correct}

the students, not the instructors, should be from the Math department

\section{Submit}

\section{Skip}

Figure 8: An example of the data collection interface. The Predicted SQL is: ' SELECT name, salary FROM instructor WHERE dept_name LIKE "math\%"'. Note that neither the gold nor the predicted SQL are shown to the annotator.

\begin{tabular}{|ll|}
\hline SQL Component & Explanation \\
\hline intersect & show the rows that are in both the results of step 1 and step 2 \\
union & show the rows that are in any of the results of step 1 and step 2 \\
except & show the rows that are in the results of step 1 but not in the results of step 2 \\
only keep the first n rows of the results in step 1 & for each row in Table 1, find corresponding rows in Table 2 \\
join $n$ & find Column of Table \\
find each value of Column1 in Table along with the OPERATION of Column2 \\
of the corresponding rows to each value \\
aggregation & $\begin{array}{l}\text { order Direction by Column } \\
\text { whose Column Operation Value } \\
\text { without repetition }\end{array}$ \\
$\begin{array}{l}\text { ordering } \\
\text { condition } \\
\text { distinct }\end{array}$ & \\
\hline
\end{tabular}

Figure 9: Examples of how different SQL components can be explained in natural language 